\title{
Semileptonic Decays with CLEO-c
}

\author{
Patrick Spradlin ${ }^{* \dagger}$ \\ University of Oxford \\ E-mail: p.spradlin1@physics.ox.ac.uk
}

This article summarizes four recent analyses of semileptonic charm and charmed-strange meson decays in data collected with the CLEO-c detector. These include an improved analysis of the partial rates of semileptonic decays of $D$ mesons to $\pi$ and $K$ mesons with fits to form factor parameterizations, a determination of the absolute branching fractions of $D_{s}$ meson decays to a set of exclusive semileptonic modes, a detailed analysis of the semileptonic decay $D_{s}^{+} \rightarrow f_{0}(980) e^{+} v_{e}$, and a measurement of the inclusive branching fractions of the decays of $D$ and $D_{s}$ mesons to semileptonic final states.

12th International Conference on B-Physics at Hadron Machines September 7-11, 2009

Heidelberg, Germany.

* Speaker.

†n behalf of the CLEO collaboration 


\section{Introduction}

Semileptonic decays are excellent processes with which to measure Standard Model (SM) parameters and to test theoretical calculations and models. This article briefly summarizes four measurements of semileptonic decays of charmed and charmed-strange mesons with data taken with the CLEO-c detector [1, 2, 3]. Section 2 introduces event reconstruction techniques common to the measurements. Section 3 describes measurements of the partial rates of the decays $D^{0} \rightarrow$ $\pi^{-} e^{+} v_{e}, D^{0} \rightarrow K^{-} e^{+} v_{e}, D^{+} \rightarrow \pi^{0} e^{+} v_{e}$, and $D^{+} \rightarrow \overline{K^{0}} e^{+} v_{e}$ in several bins of the invariant mass of the lepton-neutrino system, $q^{2}$. The measured partial rates are compared to the predictions from lattice quantum chromodynamics (LQCD) and used in fits to models of form factors $f_{+}\left(q^{2}\right)$ 陆. Section 4 summarizes the measurements of the absolute branching fractions of $D_{s}^{+}$semileptonic decays where the hadron in the final state is one of $\phi, \eta, \eta^{\prime}, K_{S}^{0}, K^{* 0}(892)$, or $f_{0}(980)$ [5]. Section 5 outlines a detailed study of the decays $D_{s}^{+} \rightarrow f_{0}(980) e^{+} v_{e}$ and $D_{s}^{+} \rightarrow \phi e^{+} v_{e}$. The decay rates are studied as functions of $q^{2}$, and the mass and width of the $f_{0}(980)$ are measured [6]. Section 6 reviews the measurements of the inclusive semileptonic branching fractions of $D^{0}, D^{+}$, and $D_{s}^{+}$ mesons [7]. Unless otherwise stated, charge conjugate modes are implied throughout this article.

\section{Event reconstruction techniques}

The measurements described in this article used two open charm data samples collected with the CLEO-c detector. Measurements of semileptonic decays of $D^{0}$ and $D^{+}$mesons (Sections 3 and (6) are based on the $\psi(3770) \rightarrow D \bar{D}$ sample. The measurements of semileptonic decays of $D_{s}^{+}$ mesons (Sections 4, 6, and 5) are based on a sample collected in $e^{+} e^{-}$collisions at a center of mass (CM) energy near $4170 \mathrm{MeV}$. At this CM energy, the cross section for $e^{+} e^{-}$annihilation into $D_{s}^{ \pm} D_{s}^{* \mp}$ is approximately $0.9 \mathrm{nb}[8]$.

Each of the measurements relied on a tagging technique in which one of the charm mesons in the event, designated the 'tag', was fully reconstructed in one of a set of decay modes. The semileptonic decay under study was reconstructed in the system recoiling from the tag. In addition to low levels of backgrounds, the resulting tagged sample has a known number of $D^{0} \overline{D^{0}}, D^{+} D^{-}$, or $D_{s}^{ \pm} D_{s}^{* \mp}$ that provides an absolute normalization for semileptonic branching fractions.

The details of the tag selection for each measurement are fully described in their respective publications [ [, , 5, 6, 7]. After basic reconstruction and particle identification criteria, backgrounds in the reconstruction of $D^{-}$and $\overline{D^{0}}$ tags were reduced with cuts on the difference between the reconstructed tag energy $E_{\text {tag }}$ and the beam energy $E_{\text {beam }}, \Delta E \equiv E_{\text {beam }}-E_{\text {tag }}$, and on the beam constrained mass, $M_{\mathrm{BC}} \equiv\left(E_{\text {beam }}^{2} / c^{4}-\left|\mathbf{P}_{\text {tag }}\right|^{2} / c^{2}\right)^{1 / 2}$, where $\mathbf{P}_{\text {tag }}$ is the reconstructed tag momentum. Figure $1 \mathrm{~A}$ shows the distributions of $M_{\mathrm{BC}}$ for the three $\overline{D^{0}}$ tag modes and the six $D^{-}$tag modes used in the partial rate measurements of $D \rightarrow K / \pi e^{+} v_{e}$ (Section 3, Ref. [ [卂]). The inclusive $D^{0}$ and $D^{+}$semileptonic branching fraction measurements each used a single very clean tag mode, $\overline{D^{0}} \rightarrow K^{+} \pi^{-}$and $D^{-} \rightarrow K^{+} \pi^{-} \pi^{-}$respectively (Section 6, Ref. [7]).

A tag $D_{s}^{-}$in the $4170 \mathrm{MeV}$ sample may have been directly produced in a $D_{s}^{*+} D_{s}^{-}$event, or it may have been the product of a $D_{s}^{*-}$ decay in a $D_{s}^{+} D_{s}^{*-}$ event. For the latter, $M_{\mathrm{BC}}$ peaks broadly due to the photon in the tag side, so it was used as a coarse selection variable. More general background discrimination was obtained by identifying candidate photons produced by $D_{s}^{* \mp}$ decays 


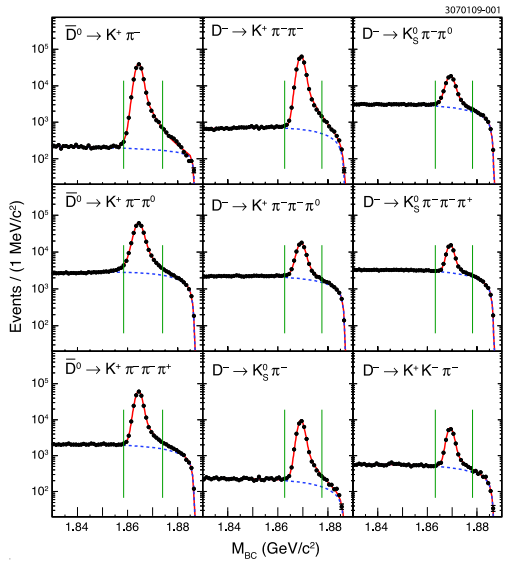

(A) Tags at $\psi(3770)$
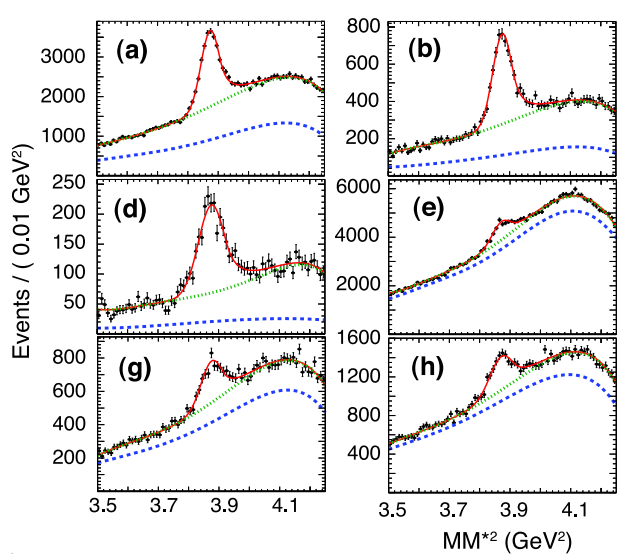

(B) Tags at $4170 \mathrm{MeV}$

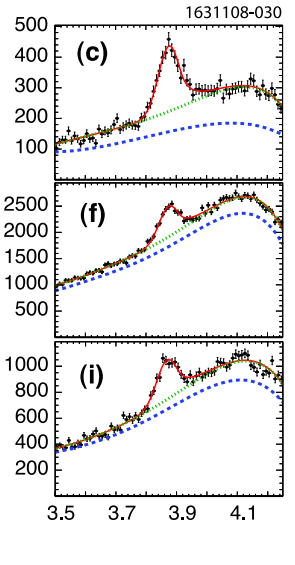

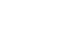

Figure 1: Mass distributions used in the measurement of tag yields. A) $D^{-}$and $\overline{D^{0}}$ tags at $\psi(3770)$. $M_{\mathrm{BC}}$ distributions in data (points), with fits (solid lines) and background contributions to fits (dotted

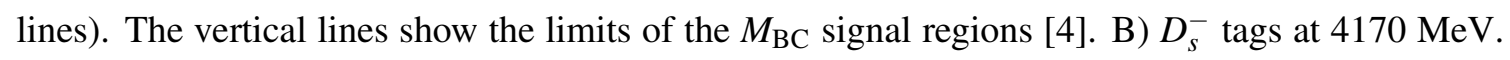
The $M M^{* 2}$ distribution from events with a photon in addition to the $D_{s}^{-}$tag for the modes: (a) $K^{+} K^{-} \pi^{-}$, (b) $K_{S}^{0} K^{-}$, (c) $\eta \pi^{-}$, (d) $\eta^{\prime} \pi^{-}$, (e) $K^{+} K^{-} \pi^{-} \pi^{0}$, (f) $\pi^{+} \pi^{-} \pi^{-}$, (g) $K^{*-}(892) K^{* 0}(892)$, (h) $\eta \rho^{-}$, (i) $\eta^{\prime} \pi^{-}, \eta^{\prime} \rightarrow \pi^{+} \pi^{-} \gamma$. The curves are fits to Crystal-Ball functions and two 5 th order Chebychev background functions [9].

and measuring the missing mass-squared recoiling against the $\gamma$-tag system $M M^{* 2}$,

$$
M M^{* 2} \equiv\left(E_{\mathrm{ee}}-E_{D_{s}}-E_{\gamma}\right)^{2}-\left(\mathbf{p}_{\mathrm{ee}}-\mathbf{p}_{D_{s}}-\mathbf{p}_{\gamma}\right)^{2}
$$

where $\left(E_{\mathrm{ee}}, \mathbf{p}_{\mathrm{ee}}\right)$ is the net four-momentum of the $e^{+} e^{-}$interaction taking the beam crossing angle into account, $\left(E_{D_{s}}, \mathbf{p}_{D_{s}}\right)$ is the four-momentum of the $\operatorname{tag} D_{s}^{-}$, and $\left(E_{\gamma}, \mathbf{p}_{\gamma}\right)$ is the four-momentum of the candidate $D_{s}^{* \mp}$ daughter photon. Regardless of whether the photon was produced with the tag or signal meson, $M M^{* 2}$ peaks at the $D_{s}^{+}$mass squared for correctly reconstructed $D_{s}^{ \pm} D_{s}^{* \mp}$ events. Figure $1 \mathrm{~B}$ shows the $M M^{* 2}$ distributions for the nine tag modes used in the $D_{s}^{+} \rightarrow f_{0}(980) e^{+} v_{e}$ study (Section 5, Ref. [6]). The absolute branching fraction measurements (Section 4 , Ref. [5]) used the same nine tag modes, but approximately half of the full data sample. The inclusive $D_{s}^{+}$ semileptonic branching fraction measurement used a single clean tag mode, $D_{s}^{-} \rightarrow \phi \pi^{-}$(Section 6 , Ref. [7]).

\section{Partial rates of $D$ meson semileptonic decays to $\pi$ and $K$}

We measured the partial rates of the four charm decays $D^{0} \rightarrow \pi^{-} e^{+} v_{e}, D^{0} \rightarrow K^{-} e^{+} v_{e}, D^{+} \rightarrow$ $\pi^{0} e^{+} v_{e}$, and $D^{+} \rightarrow \overline{K^{0}} e^{+} v_{e}$ as functions of $q^{2}$ [ [П]. For semileptonic decays in which the initial and final state hadrons are pseudoscalars and the lepton mass is negligibly small, the strong interaction dynamics can be described by a single form factor $f_{+}\left(q^{2}\right)$. The rate for a $D$ semileptonic decay with final state meson $P$ is given by

$$
\frac{d \Gamma\left(D \rightarrow P e v_{e}\right)}{d q^{2}}=X \frac{G_{F}^{2}\left|V_{c d(s)}\right|^{2}}{24 \pi^{3}} p^{3}\left|f_{+}\left(q^{2}\right)\right|^{2},
$$




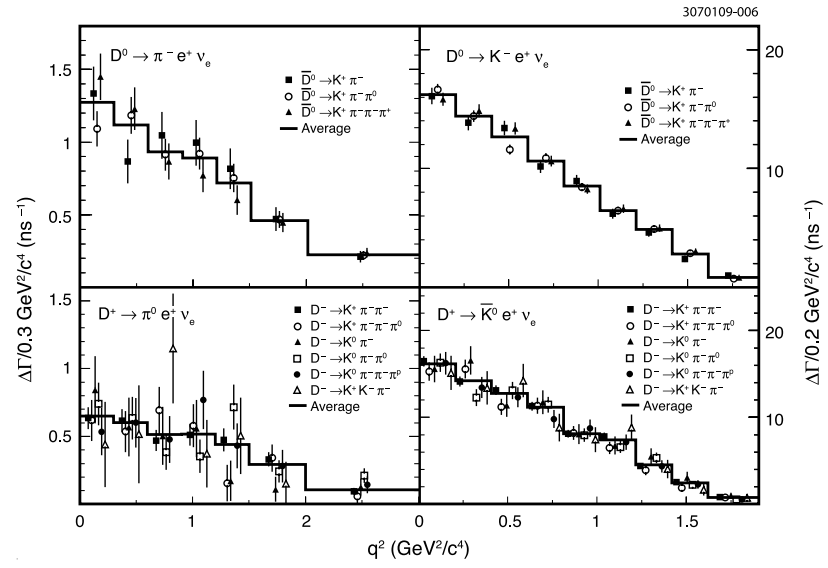

Figure 2: Partial rates for each semileptonic mode. The points show measurements in each tag mode; the histograms show the partial rates averaged over all tag modes [ $₫$.

where $G_{F}$ is the Fermi constant, $V_{c d(s)}$ is the relevant CKM matrix element, $p$ is the momentum of the daughter meson in the rest frame of the parent $D$, and $X$ is a multiplicative factor due to isospin. The isospin factor $X$ in Equation 3.1 is equal to 1 for each of the modes except $D^{+} \rightarrow \pi^{0} e^{+} v_{e}$, where it is $1 / 2$.

In the full $818 \mathrm{pb}^{-1}$ CLEO-c $\psi(3770)$ data set, we selected tagged events by reconstructing three $\overline{D^{0}}$ tag modes and the six $D^{-}$tag modes as described in Section 2. Semileptonic signal candidates were formed from positron and hadron candidate pairs in the system recoiling against the tag. Although the neutrino daughter is not detected, its energy and momentum, and hence $q^{2}$, can be inferred from the missing four-momentum of the event $\left(E_{\text {miss }}, \mathbf{P}_{\text {miss }}\right)$. We partitioned the candidates into bins of $q^{2}$ and extracted the signal yields independently for each $q^{2}$ bin and tag mode with a fit to the variable $U \equiv E_{\text {miss }}-c\left|\mathbf{P}_{\text {miss }}\right|$, which peaks at zero for correctly reconstructed signal events. Figure 2 shows the resulting partial rates for each tag mode and averaged over the tag modes.

To extract form factor parameters, branching fractions, and the magnitudes of CKM elements $V_{c d}$ and $V_{c s}$ we fit the partial rate results using Equation 3.1 and a parameterization of the form factor $f_{+}\left(q^{2}\right)$. We performed $\chi^{2}$ fits using each of five parameterizations: the simple pole model [10], the modified pole model [10], the series expansion of [11] carried out to first order in the expansion variable $z\left(q^{2}, t_{0}\right)$, the series expansion carried out to second order in $z\left(q^{2}, t_{0}\right)$, and the ISGW2 model [12]. We took the second order series expansion as our nominal fit for further analysis. The fits with the other parameterizations test the underlying assumptions of the respective models and facilitate comparisons of our results with previous analyses. As long as the normalization $\left|V_{c d(s)}\right| f_{+}(0)$ and at least one shape parameter were allowed to float, all models described the data well. Integrating our nominal fit over $q^{2}$ we obtained branching fractions for each mode

$$
\begin{aligned}
& \mathscr{B}\left(D^{0} \rightarrow \pi^{-} e^{+} v_{e}\right)=(0.288 \pm 0.008 \pm 0.003) \% \\
& \mathscr{B}\left(D^{0} \rightarrow K^{-} e^{+} v_{e}\right)=(3.50 \pm 0.03 \pm 0.04) \% \\
& \mathscr{B}\left(D^{+} \rightarrow \pi^{0} e^{+} v_{e}\right)=(0.405 \pm 0.016 \pm 0.009) \%
\end{aligned}
$$



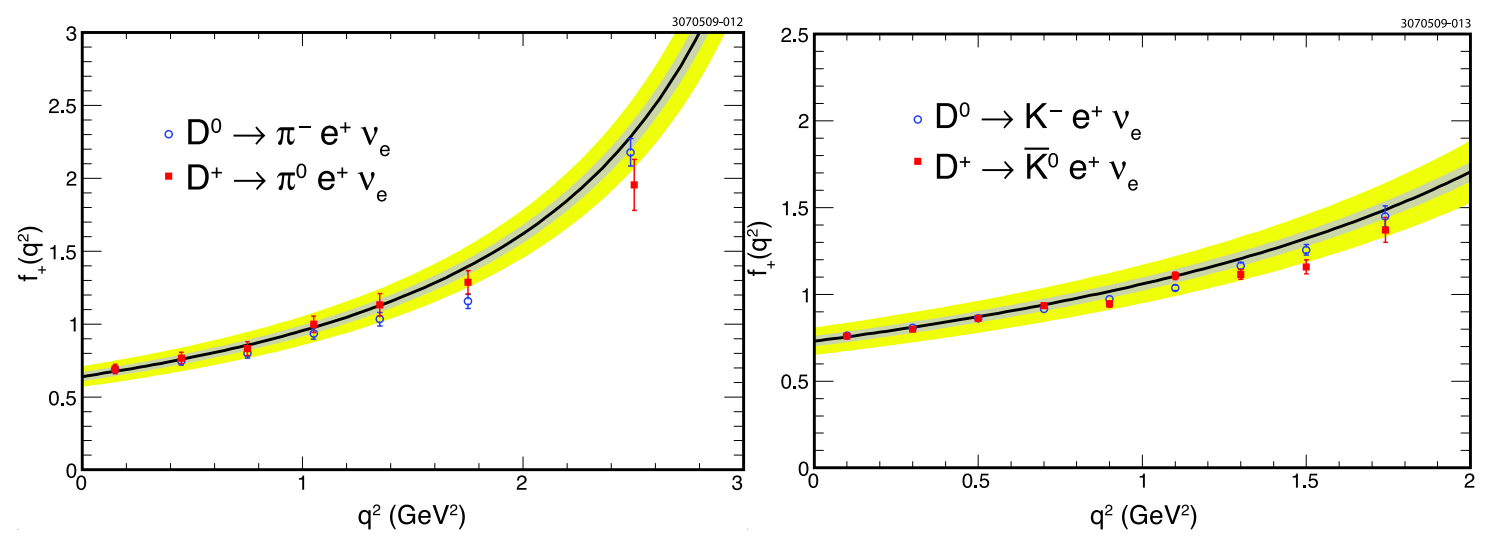

Figure 3: $f_{+}\left(q^{2}\right)$ comparison between isospin conjugate modes and with LQCD calculations 113, [14]. The solid lines represent LQCD fits to the modified pole model [10]. The inner bands show LQCD statistical uncertainties, and the outer bands the sum in quadrature of LQCD statistical and systematic uncertainties [4].

$$
\text { and } \mathscr{B}\left(D^{+} \rightarrow \overline{K^{0}} e^{+} v_{e}\right)=(8.83 \pm 0.10 \pm 0.20) \%,
$$

where the first uncertainty is statistical and the second is systematic.

If isospin is an exact symmetry, the form factors for $D^{0} \rightarrow \pi^{-} e^{+} v_{e}$ and $D^{+} \rightarrow \pi^{0} e^{+} v_{e}$ are expected to be identical, as are those for $D^{0} \rightarrow K^{-} e^{+} v_{e}$ and $D^{+} \rightarrow \overline{K^{0}} e^{+} v_{e}$. We performed combined second order series fits to these isospin conjugate pairs and extracted magnitudes of the CKM matrix elements $\left|V_{c d}\right|$ and $\left|V_{c s}\right|$ from the resulting $\left|V_{c d(s)}\right| f_{+}(0)$. Using LQCD measurements of $f_{+}(0)[13$, 14], we found

$$
\begin{aligned}
\left|V_{c d}\right| & =0.234 \pm 0.007 \pm 0.002 \pm 0.025 \\
\text { and } \quad\left|V_{c s}\right| & =0.985 \pm 0.009 \pm 0.006 \pm 0.103
\end{aligned}
$$

where the third uncertainties are from $f_{+}(0)$. These are in agreement with those reported by the Particle Data Group based on the assumption of CKM unitarity [15]. Lastly, we obtained $f_{+}\left(q^{2}\right)$ at the center of each of our $q^{2}$ bins using the Particle Data Group values for $\left|V_{c d(s)}\right|$ and compared these to the results of LQCD calculations [13, 4 . Figure 3 shows that they are in good agreement, but that our measurements have significantly smaller uncertainties.

\section{Absolute branching fraction measurements for exclusive $D_{s}$ semileptonic decays}

We measured the absolute branching fractions of $D_{s}^{+}$semileptonic decays where the hadron in the final state was one of $\phi, \eta, \eta^{\prime}, K_{S}^{0}, K^{* 0}(892)$, or $f_{0}(980)$ [5]. Tagged events were selected in a data sample of $310 \mathrm{pb}^{-1}$ collected at a CM energy near $4170 \mathrm{MeV}$ by reconstructing nine $D_{s}^{-}$tag modes as described in Section 2. For any given tag-photon combination, we sought a candidate $e^{+}$ and a candidate from the set of hadrons in the recoiling system. In each case we required that the event had no unused tracks, and that the tag and semileptonic candidate had opposite charge.

For each $\gamma$ candidate in each event, we performed two kinematic fits, one assuming that the $\gamma$ combines with the tag to form a $D_{s}^{*-}$, the other assuming that the semileptonic decay comes from 


\begin{tabular}{lr|lr}
\hline \hline Signal Mode & $\mathscr{B}(\%)$ & Signal Mode & $\mathscr{B}(\%)$ \\
\hline$D_{s}^{+} \rightarrow \phi e^{+} v_{e}$ & $2.29 \pm 0.37 \pm 0.11$ & $D_{s}^{+} \rightarrow K^{0} e^{+} v_{e}$ & $0.37 \pm 0.10 \pm 0.02$ \\
$D_{s}^{+} \rightarrow \eta e^{+} v_{e}$ & $2.48 \pm 0.29 \pm 0.13$ & $D_{s}^{+} \rightarrow K^{* 0}(892) e^{+} v_{e}$ & $0.18 \pm 0.07 \pm 0.01$ \\
$D_{s}^{+} \rightarrow \eta^{\prime} e^{+} v_{e}$ & $0.91 \pm 0.33 \pm 0.05$ & $D_{s}^{+} \rightarrow f_{0}(980) e^{+} v_{e}$ & $0.13 \pm 0.04 \pm 0.01$ \\
\hline \hline
\end{tabular}

Table 1: The derived branching fractions including the systematic errors for the six semileptonic channels studied. The $D_{s}^{+} \rightarrow f_{0}(980) e^{+} v_{e}$ branching fraction quoted represents the product branching fraction $\mathscr{B}\left(D_{s}^{+} \rightarrow f_{0}(980) e^{+} v_{e}\right) \times \mathscr{B}\left(f_{0}(980) \rightarrow \pi^{+} \pi^{-}\right)$, which is the dominant decay mode in [15].

a $D_{s}^{*+}$ parent. We chose the photon and hypothesis with the smallest $\chi^{2}$ and calculated the event missing mass squared $M M^{2}$ defined as

$$
M M^{2}=\left(E_{\mathrm{CM}}^{*}-E_{D_{s}}^{*}-E_{\gamma}^{*}-E_{e}^{*}-E_{\mathrm{had}}^{*}\right)^{2}-\left(-\mathbf{p}_{D_{s}}^{*}-\mathbf{p}_{\gamma}^{*}-\mathbf{p}_{e}^{*}-\mathbf{p}_{\mathrm{had}}^{*}\right)^{2},
$$

where $\left(E_{e}^{*}, \mathbf{p}_{e}^{*}\right)$ is the four-momentum of the positron candidate and $\left(E_{\mathrm{had}}^{*}, \mathbf{p}_{\text {had }}^{*}\right)$ is the four-momentum of the hadron candidate in the CM system. For signal events, $M M^{2}$ is the $v_{e}$ invariant mass squared and thus it peaks at zero. The signal yield $n_{\alpha, \text { sig }}^{i}$ were determined for each signal decay $i$ and each tag mode $\alpha$ by subtracting the background under the signal peak in $\left|M M^{2}\right|<0.05 \mathrm{GeV}^{2}$. We evaluated exclusive branching fractions from $n_{\alpha, \text { sig }}^{i}$ through the relationship

$$
\mathscr{B}_{i}=\frac{\sum_{\alpha} n_{\alpha, \mathrm{sig}}^{i}}{\varepsilon_{\mathrm{SL}}^{i}\left(\sum_{\alpha} n_{\alpha}\right) \mathscr{B}_{i}^{\text {had }}},
$$

where $\varepsilon_{\mathrm{SL}}^{i}$ represents the average efficiency for finding the exclusive semileptonic decay in the tag sample used (estimated from Monte Carlo), $n_{\alpha}$ is the number of tags in mode $\alpha$, and $\mathscr{B}_{i}^{\text {had }}$ is the branching fraction for the final state in which the hadron $i$ was reconstructed. The resulting absolute branching fraction measurements with statistical and systematic uncertainties appear in Table 1 .

\section{Semileptonic decays $D_{s}^{+} \rightarrow f_{0}(980) e^{+} v_{e}$ and $D_{s}^{+} \rightarrow \phi e^{+} v_{e}$}

We studied the semileptonic decays $D_{s}^{+} \rightarrow f_{0}(980) e^{+} v_{e}$ and $D_{s}^{+} \rightarrow \phi e^{+} v_{e}$ in more detail with the full $600 \mathrm{pb}^{-1}$ CLEO-c data set near CM energy $4170 \mathrm{MeV}$ [6]. Our measurements include improved branching fractions, a form factor fit for the decay $D_{s}^{+} \rightarrow f_{0}(980) e^{+} v_{e}$, and a measurement of the mass and width of $f_{0}(980)$. Section 2 describes the selection of tags. The final states $f_{0}(980) e^{+} v_{e}$ and $\phi e^{+} v_{e}$ were reconstructed opposite to these tags with selection criteria similar to those for the absolute branching fraction measurements of Section 4

For the form factor measurements, we combined the tag modes and separated the data into five $q^{2}$ bins on which we performed fits to the mass distributions. After correcting the yields for reconstruction efficiencies, we fit the resulting $f_{0}(980) e^{+} v_{e}$ partial rates using a modified form of Equation 3.1 to account for the finite width of the $f_{0}(980)$ [16]. A simple pole model was assumed for $f_{+}\left(q^{2}\right)$. The fit appears in Figure $4 \mathrm{a}$, and the estimated pole mass is $\left(1.7_{-0.7}^{+4.5} \pm 0.2\right) \mathrm{GeV}$. For $\phi e^{+} v_{e}$, we used fixed form factors measured by BABAR [17], and fit to determine independently 


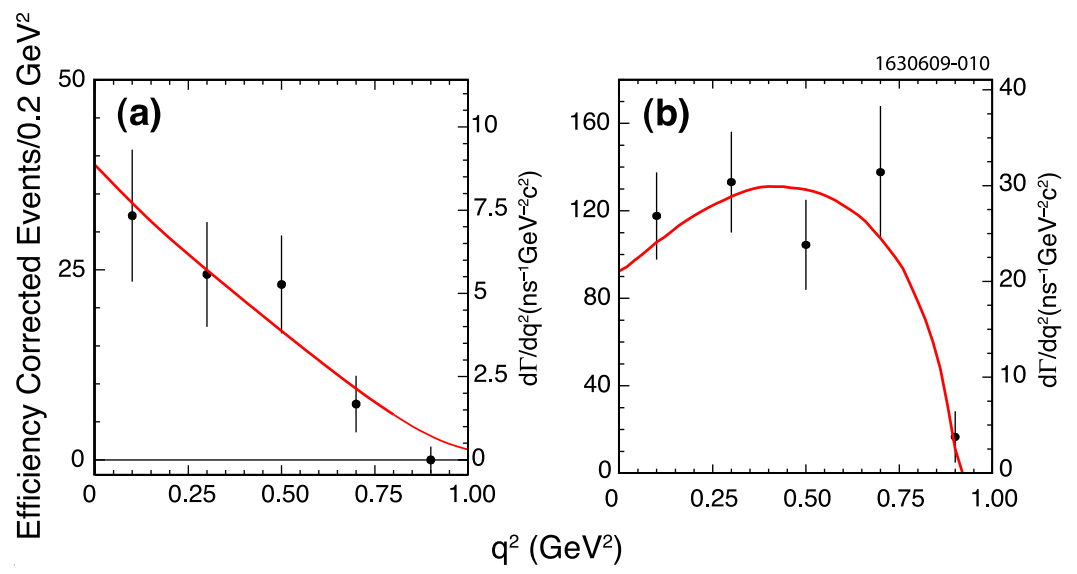

Figure 4: The $q^{2}$ distributions for (a) $D_{s}^{+} \rightarrow f_{0}(980) e^{+} v_{e}$ fit to a function allowing a varying pole mass and (b) $D_{s}^{+} \rightarrow \phi e^{+} v_{e}$ fit to a function with form factors fixed to those measured by BABAR [17] [6].

our decay rate at $q^{2}$ equal to zero (Figure $\bigoplus \mathrm{b}$ ):

$$
\frac{\left.\frac{d \Gamma}{d q^{2}}\left(D_{s}^{+} \rightarrow f_{0}(980) e^{+} v_{e}, f_{0}(980) \rightarrow \pi^{+} \pi^{-}\right)\right|_{q^{2}=0}}{\left.\frac{d \Gamma}{d q^{2}}\left(D_{s}^{+} \rightarrow \phi e^{+} v_{e}, \phi \rightarrow K^{+} K^{-}\right)\right|_{q^{2}=0}}=(42 \pm 11) \% .
$$

Stone and Zhang to have predicted that this ratio is equal to the ratio $\Gamma\left(B_{s} \rightarrow J / \psi f_{0}(980), f_{0}(980) \rightarrow\right.$ $\left.\pi^{+} \pi^{-}\right) / \Gamma\left(B_{s} \rightarrow J / \psi \phi, \phi \rightarrow K^{+} K^{-}\right)$[18]. Our measurement is encouraging for the prospects of $C P$ violation measurements with $B_{s} \rightarrow J / \psi f_{0}(980)$.

Our updated absolute branching fraction measurement for $D_{s}^{+} \rightarrow f_{0}(980) e^{+} v_{e}$ is

$$
\mathscr{B}\left(D_{s}^{+} \rightarrow f_{0}(980) e^{+} v_{e}\right) \mathscr{B}\left(f_{0}(980) \rightarrow \pi^{+} \pi^{-}\right)=(0.20 \pm 0.03 \pm 0.01) \%,
$$

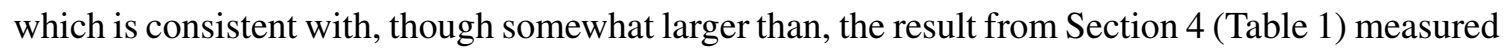
with half of the amount of data. Taking a $\phi \rightarrow K^{+} K^{-}$branching fraction of $(49.2 \pm 0.6) \%$ [15], we obtained

$$
\mathscr{B}\left(D_{s}^{+} \rightarrow \phi e^{+} v_{e}\right)=(2.36 \pm 0.23 \pm 0.13) \%,
$$

which is consistent with the previous result (Table 1). Finally, we fit the $\pi^{+} \pi^{-}$invariant mass distribution for the collected $f_{0}(980) e^{+} v_{e}$ data with a relativistic Breit-Wigner function modified by phase space effects to measure the mass and width of $f_{0}(980)$ to be

$$
\begin{aligned}
M_{f_{0}(980)} & =\left(977_{-9}^{+11} \pm 1\right) \mathrm{MeV}, \\
\text { and } \quad \Gamma_{0} & =\left(91_{-22}^{+30} \pm 3\right) \mathrm{MeV}
\end{aligned}
$$

\section{Inclusive semileptonic decays of charm and charmed-strange mesons}

We used the complete CLEO-c open charm data sets, $818 \mathrm{pb}^{-1}$ near the $\psi(3770)$ resonance and $602 \mathrm{pb}^{-1}$ at $\mathrm{CM}$ energy $4170 \mathrm{MeV}$, to measure the inclusive semileptonic branching fractions 

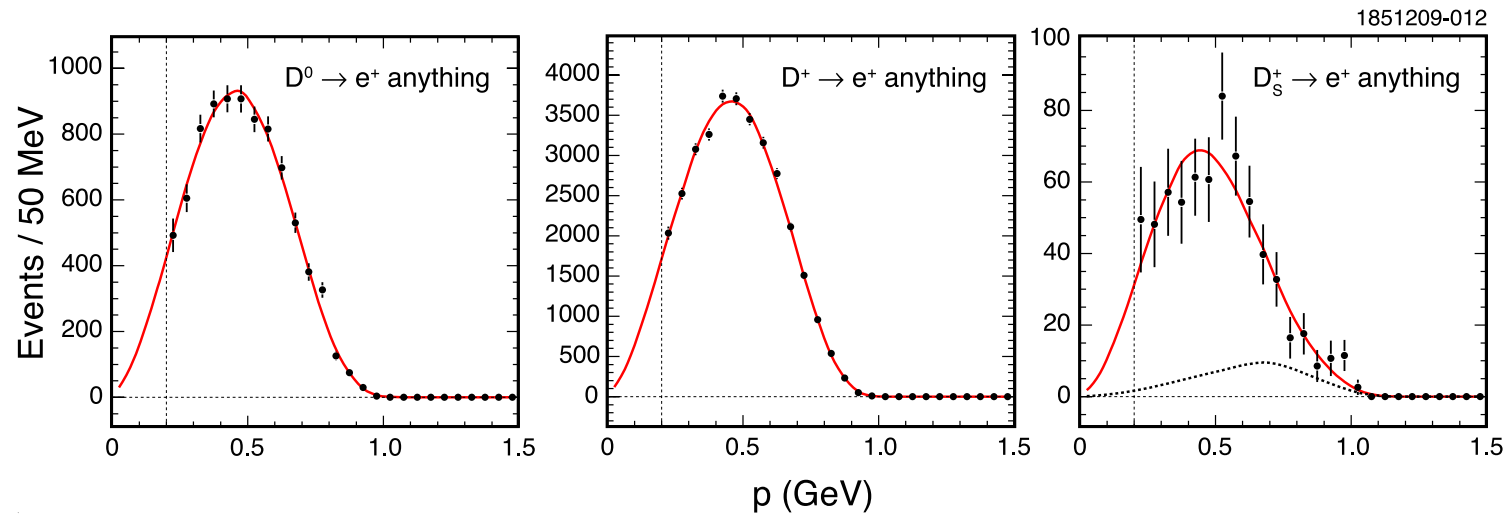

Figure 5: Inclusive laboratory frame electron spectra obtained from data, shown as points with statistical uncertainties. The vertical dashed lines indicate the PID momentum cutoff at $200 \mathrm{MeV}$. Extrapolated spectra are shown as solid curves. The dashed curve in the $D_{s}^{+}$spectrum plot is the expected contribution from $\tau^{+} \rightarrow e^{+} v_{e} \bar{v}_{\tau}$ from leptonic $D_{s}^{+} \rightarrow \tau^{+} v_{\tau}$ decays [7].

$D^{0} \rightarrow X e^{+} v_{e}, D^{+} \rightarrow X e^{+} v_{e}$, and $D_{s}^{+} \rightarrow X e^{+} v_{e}$ [7]. As described in Section \&, three very clean single tag modes composed of only charged particles were used: $\overline{D^{0}} \rightarrow K^{+} \pi^{-}, D^{-} \rightarrow K^{+} \pi^{-} \pi^{-}$, and $D_{s}^{-} \rightarrow \phi \pi^{-}$.

Double tag events for further analysis were identified by adding a recoiling charged track opposite to the reconstructed tag. We required the momentum of the track to be $p>200 \mathrm{MeV}$ and the angle with respect to the beam to be $|\cos \theta|<0.80$ so that all charged-particle identification (PID) information $(d E / d x, \mathrm{RICH}$, and $E / p)$ was available. The track was also required to be identified as an electron, a charged pion, or a charged kaon.

The $D$ or $D_{s}$ semileptonic inclusive spectrum (or differential decay rate) can be measured as

$$
\frac{1}{\Gamma} \frac{d \Gamma_{\mathrm{SL}}}{d p}=\frac{1}{n_{D}} \frac{\Delta n}{\Delta p}=\frac{1}{n_{\mathrm{ST}}} \frac{\Delta y / \varepsilon_{\mathrm{SL}}}{\Delta p},
$$

where $n_{D}$ is the number of $D$ mesons produced, $n$ is the number of primary electrons in bins of momentum $p, n_{\mathrm{ST}}$ is the number of single tags (ST), $y$ is the electron candidate yield in double tag events in bins of momentum, and $\varepsilon_{\mathrm{SL}}$ is the electron detection efficiency. We measured the yields $y$ with a generalized unfolding method that corrects for resolution and misidentification effects. After additional corrections for doubly Cabibbo-suppressed tag decays in the $D^{0} \rightarrow X e^{+} v_{e}$ analysis, for the expected contributions from $D_{(s)}^{+} \rightarrow \tau^{+} v_{\tau}, \tau^{+} \rightarrow e^{+} v_{e} \bar{v}_{\tau}$ in $D^{+} \rightarrow X e^{+} v_{e}$ and $D_{s}^{+} \rightarrow X e^{+} v_{e}$, and for possible biases produced by the double tagging method in all modes, we obtained the inclusive laboratory frame semileptonic spectra show in Figure 5. The curves used to extrapolate the spectra below the $200 \mathrm{MeV}$ momentum cutoff were obtained with a fit using the sum of measurements of exclusive channels together with form-factor models and adding higherresonance and non-resonant channels to match the sum of the exclusive channels with our observed inclusive branching fraction.

Our measured inclusive semileptonic branching fractions for charm and charmed-strange mesons are

$$
\mathscr{B}\left(D^{0} \rightarrow X e^{+} v_{e}\right)=(6.46 \pm 0.09 \pm 0.11) \%
$$




$$
\begin{aligned}
\mathscr{B}\left(D^{+} \rightarrow X e^{+} v_{e}\right) & =(16.13 \pm 0.10 \pm 0.29) \% \\
\text { and } \quad \mathscr{B}\left(D_{s}^{+} \rightarrow X e^{+} v_{e}\right) & =(6.52 \pm 0.39 \pm 0.15) \%
\end{aligned}
$$

Using known [15] lifetimes $\tau_{D^{0}}, \tau_{D^{+}}$, and $\tau_{D_{s}^{+}}$we obtained the ratios of semileptonic decay widths

$$
\begin{aligned}
& \frac{\Gamma\left(D^{+} \rightarrow X e^{+} v_{e}\right)}{\Gamma\left(D^{0} \rightarrow X e^{+} v_{e}\right)}=0.985 \pm 0.015 \pm 0.024 \\
\text { and } \quad & \frac{\Gamma\left(D_{s}^{+} \rightarrow X e^{+} v_{e}\right)}{\Gamma\left(D^{0} \rightarrow X e^{+} v_{e}\right)}=0.828 \pm 0.051 \pm 0.025
\end{aligned}
$$

The first ratio shows that charged and neutral charm meson semileptonic decay widths are consistent with isospin symmetry. The second indicates that there is a difference between charm and charmed-strange mesons semileptonic decay widths.

\section{Conclusion}

Quantum correlated production of charm and charmed-strange mesons at CLEO-c provide excellent conditions under which to measure properties of semileptonic decays and test the predictions of LQCD. This article has reviewed four of our recent results with this superb data set.

\section{References}

[1] CLEO Collaboration, Y. Kubota et al., The CLEO-II detector, Nucl. Instrum. Meth. A320 (1992) 66-113.

[2] D. Peterson et al., The CLEO III drift chamber, Nucl. Instrum. Meth. A478 (2002) 142-146.

[3] M. Artuso et al., The CLEO RICH detector, Nucl. Instrum. Meth. A554 (2005) 147-194 [arXiv:physics/0506132].

[4] CLEO Collaboration, D. Besson et al., Improved measurements of D meson semileptonic decays to $\pi$ and K mesons, Phys. Rev. D80 (2009) 032005 [arXiv: 0906.2983 [hep-ex] ] .

[5] CLEO Collaboration, J. Yelton et al., Absolute branching fraction measurements for exclusive $D_{s}$ semileptonic decays, Phys. Rev. D80 (2009) 052007 [arXiv: 0903.0601 [hep-ex] ] .

[6] CLEO Collaboration, K. M. Ecklund et al., Study of the semileptonic decay $D_{s}^{+} \rightarrow f_{0}(980) e^{+} v$ and implications for $B_{s} \rightarrow J / \psi f_{0}$, Phys. Rev. D80 (2009) 052009 [arXiv: 0907.3201 [hep-ex] ] .

[7] CLEO Collaboration, D. M. Asner et al., Inclusive semileptonic decays of charm and charmed-strange mesons, Submitted to Phys. Rev. D (2009) [arXiv:0912.4232 [hep-ex] ].

[8] CLEO Collaboration, D. Cronin-Hennessy et al., Measurement of charm production cross sections in $e^{+} e^{-}$annihilation at energies between 3.97 and $4.26 \mathrm{GeV}$, Phys. Rev. D80 (2009) 072001 [arXiv:0801.3418 [hep-ex]].

[9] CLEO Collaboration, J. P. Alexander et al., Measurement of $\mathscr{B}\left(D_{s}^{+} \rightarrow \ell^{+} v\right)$ and the decay constant $f_{D_{s}^{+}}$from $600 \mathrm{pb}^{-1}$ of $e^{+} e^{-}$annihilation data near $4170 \mathrm{MeV}$, Phys. Rev. D79 (2009) 052001 [arXiv:0901.1216 [hep-ex]].

[10] D. Becirevic and A. B. Kaidalov, Comment on the heavy $\rightarrow$ light form factors, Phys. Lett. B478 (2000) 417-423 [arXiv: hep-ph/9904490]. 
[11] T. Becher and R. J. Hill, Comment on form-factor shape and extraction of $\left|V_{u b}\right|$ from $B \rightarrow \pi \ell v$, Phys. Lett. B633 (2006) 61-69 [arXiv : hep-ph/ 0509090 ] .

[12] D. Scora and N. Isgur, Semileptonic meson decays in the quark model: An update, Phys. Rev. D52 (1995) 2783-2812 [arXiv: hep-ph/9503486].

[13] Fermilab Lattice, MILC, and HPQCD Collaborations, C. Aubin et al., Semileptonic Decays of D Mesons in Three-Flavor Lattice QCD, Phys. Rev. Lett. 94 (2005) 011601 [arXiv:hep-ph/0408306].

[14] Fermilab Lattice and MILC Collaborations, C. Bernard et al., Visualization of semileptonic form factors from lattice QCD, Phys. Rev. D80 (2009) 034026 [arXiv:0906.2498 [hep-lat] ] .

[15] Particle Data Group Collaboration, C. Amsler et al., Review of Particle Physics, Phys. Lett. B667 (2008) 1.

[16] N. Isgur, D. Scora, B. Grinstein, and M. B. Wise, Semileptonic B and D decays in the quark model, Phys. Rev. D39 (1989) 799-818.

[17] BABAR Collaboration, B. Aubert et al., Study of the decay $D_{s}^{+} \rightarrow K^{+} K^{-} e^{+} v_{e}$, Phys. Rev. D78 (2008) 051101 [arXiv:0807.1599 [hep-ex] ].

[18] S. Stone and L. Zhang, S-waves and the measurement of CP violating phases in $B_{s}$ decays, Phys. Rev. D79 (2009) 074024 [arXiv:0812.2832 [hep-ph] ]. 